\title{
Non-Equilibrium Molecular Dynamics Simulation of Gas Flow in Organic Nanochannels
}

\author{
Mohammad Kazemi Ali Takbiri-Borujeni
}

April 19, 2016

\begin{abstract}
The objective for this work is to investigate the contribution of the adsorbed phase to the mass flux and comparing transport of gases with different adsorption affinities in organic nano-scale channels. In this work, force-driven Non-Equilibrium molecular dynamics (NEMD) simulations are used to compare the transport of gases with high adsorption affinity (Methane and Argon) with the ones with low adsorption affinity (Helium), for channel heights of 2, 4, 6, and 8 nanometers at two Knudsen numbers of 0.1 and 0.2. Velocity and mass flux profiles across the channel for Argon, Methane, and Helium are compared. Transport diffusion coefficients and molecular flux of these gas are also calculated. Furthermore, adsorption properties are analysed using Grand Canonical Monte Carlo simulations.

For all the gases studied, plug-shaped velocity profiles are observed irrespective of the channel size and Knudsen number. Mass flux profiles of Argon and Methane across the channels demonstrate a significant contribution of adsorbed molecules to the total mass flux. Furthermore, as Knudsen number increases, the contribution of the adsorbed phase to the total mass flux becomes higher. Molecular flux of Helium is smaller than that of Argon and Methane for all channel sizes. The calculated diffusion coefficients of Methane are higher than those for Argon for all the channel sizes and they decrease as the channel size increases. For Argon and Methane, the diffusion coefficients become smaller as Knudsen number increases. For Helium, the diffusion coefficients are weak functions of the channel size and Knudsen number. Based on the results, contribution of the adsorbed molecules can be more than $50 \%$ of the total mass flux of the channel. For the pressure ranges studies, transport diffusivity of Helium is less sensitive to pressure and Knudsen number compared to Argon and Methane.

This study shows that the transport through organic nano-scale conduits is essentially diffusive. Therefore, to have a realistic model for predicting the recovery of fluids from unconventional resources, the transport equations in organic nanopores should be replaced by the diffusive transport equations.
\end{abstract}

\section{Introduction}

Gas recovery mechanisms from shale resources are different from those of conventional resources due to presence of organic nanopores. In one hand, the gas molecules have a tendency to be adsorbed to the surfaces of the organic pores that results in heterogeneous fluid distributions within the pores. Adsorption of gas molecules to the pore surfaces causes molecular level transport effects, such as surface-diffusion. On the other hand, the pore sizes are comparable to the mean free path of the molecules, which causes noncontinuum flow regimes. The transport of fluids in this systems cannot be described by classical continuumbased hydrodynamic equations. A question then arises as to how different is the transport of gases with low

(C) 2016. This manuscript version is made available under the Elsevier user license http://www.elsevier.com/open-access/userlicense/1.0/ 
adsorption affinity, e.g., Helium, compared to the ones with high adsorption affinities, e.g., Methane and Argon, in organic nano-scale conduits? Answer to this question can enhance our understanding of the fluid transport mechanisms in shale resources.

Molecular dynamics is an effective tool to study the details of fluid-fluid and fluid-solid interactions in nano-scale conduits. Transport of gas molecules in microporous systems have been extensively studied using three different MD simulation methods: equilibrium molecular dynamics (EMD), external field NonEquilibrium Molecular Dynamics (NEMD), and boundary driven non-equilibrium molecular dynamics (dual control volume grand canonical molecular dynamics or DCV-GCMD)(Arya et al., 2001).

DCV-GCMD simulations have been used to investigate the sensitivity of transport properties of methane, carbon dioxide, and nitrogen to pore size, porosity, and pressure gradient (Firouzi and Wilcox, 2012). Jin and Firoozabadi (2015) investigated flow of Methane in carbon nanotubes using DCV-GCMD simulations and the mobility of adsorbed phase in high and low pressures were demonstrated. They found that the Knudsen diffusion and Hagen-Poiseuille equations can underestimate the Methane flow by one order of magnitude. Kazemi and Takbiri-Borujeni (2016b) studied the transport of four different gases, Methane and Argon (adsorbing) and Helium and Neon (less-adsorbing). They showed that as the average channel pressure increases, the contribution of the adsorbed phase to the total mass flux decreases. For organic nanocapillaries, DCV-GCMD simulations have shown that the Knudsen diffusion model underestimates the molecular flux Kazemi and Takbiri-Borujeni (2016a). This underestimation was not a strong function of the pore size or the pore pressure.

NEMD simulations are easy to implement and computationally efficient for simulating the transport phenomena; however, the equivalence of external forcing function that drives diffusion and the actual chemical potential gradient has not been formally demonstrated (Arya et al., 2001). NEMD simulations have been applied to investigate the slippage of a non-adsorbing gas (Helium) in microporous media (Firouzi et al., 2014). Collell et al. (2015) performed MD simulations of hydrocarbons flowing through a molecular model of kerogen type II. The results suggested that the flow inside kerogen is of diffusive nature. Feng et al. (2015) carried out NEMD and DCV-GCMD to study the flow of Methane in nano-capillaries. They showed that the surface transport of adsorbed Methane have a significant contribution to total mass flux in nano-capillaries.

Comparing the transport properties of different types of gases in nano-scale conduits using NEMD is challenging because these systems have many degrees of freedom, e.g., channel size, molecule size, gas density, and therefore, finding equivalent systems is difficult. Furthermore, for systems under confinement, there is a positional dependence of density and also the state variables, pressure and temperature (Travis et al., 2000). Investigation of gas systems with respect to the Knudsen number might be a viable option. The gas flow regimes are characterized by Knudsen number $(K n)$, which is the ratio of mean free path of molecules to the characteristic length of the system,

$$
K n=\frac{1}{\sqrt{2} \pi n H d^{2}},
$$

where $n$ is the number density, $H$ is the channel height, and $d$ is the molecular diameter. This formulation of Knudsen number incorporates molecular size, number density, and channel size and provides a means for comparing systems with different sizes and gas types.

The objective for this work is to investigate the adsorbed phase transport by comparing the velocity profiles, mass fluxes, and diffusion coefficients of different gases with different adsorption affinities (Argon, Methane, and Helium). In order to determine the adsorption affinities of different gases, Grand Canonical Monte Carlo 
simulations are performed for two channel heights of 2 and $4 \mathrm{~nm}$. NEMD simulations of force-driven flow is carried out in graphite nanochannels with 2, 4, 6, and $8 \mathrm{~nm}$ height at Knudsen numbers of 0.1 and 0.2 . The density, velocity profiles, mass fluxes, and diffusion coefficients of these gases are then compared.

\section{Molecular Dynamics Methods}

The Grand Canonical Monte Carlo (GCMC) simulations are performed at different pressures for two graphite nanochannel heights of 2 and $4 \mathrm{~nm}$ to determine the gas adsorption isotherms of Methane, Argon, and Helium. In GCMC simulations, the pressure inside the control volume is kept constant by insertion and deletion of molecules. The probability of inserting a molecule is determined as,

$$
p^{+}=\min \left\{\frac{Z V_{C V}}{N_{C V}+1} \exp \left(-\frac{\Delta U}{k_{B} T}\right), 1\right\}
$$

where $Z=\exp \left(\frac{\mu}{k_{B} T}\right) / \Lambda$ is the absolute activity at temperature $T, \Lambda$ is the de Broglie wavelength, $\mu$ is the chemical potential, and $k_{B}$ is the Boltzmann constant. Changes in potential energy resulting from insertion and deletion of molecules are represented by $\Delta U$, volume of control volume is $V_{C V}$, and number of molecules in control volume is $N_{C V}$. Inserted molecules are assigned a velocity using Maxwell-Boltzmann distribution. The probability of deleting a molecule is,

$$
p^{-}=\min \left\{\frac{N_{C V}}{Z V_{C V}} \exp \left(-\frac{\Delta U}{k_{B} T}\right), 1\right\}
$$

The temperature of the inserted molecules is determined based on the specified reservoir temperature (300 $\mathrm{K})$.

Non-equilibrium molecular dynamics (NEMD) simulations of force-driven flow of Argon, Methane, and Helium are performed in three-dimensional graphite channels (with hexagonal lattice) of heights 2, 4, 6, 8 nanometers $(\mathrm{nm})$. The width of the channel is chosen to be $4.5 \mathrm{~nm}$ for all the simulations; however, the channel lengths change with respect to the channel height such that the ratio of the length to height of the channels to be 20 in order to have a fully developed flow. For example, the channel lengths for simulations with $2 \mathrm{~nm}$ and $4 \mathrm{~nm}$ channel heights is selected to be $40 \mathrm{~nm}$ and $80 \mathrm{~nm}$, respectively. The three-layered channel walls are placed in $\mathrm{x}-\mathrm{y}$ plane. The simulations are performed for two Knudsen numbers of 0.1 and 0.2. The number of molecules for each specific gas and geometry is different and is determined by Eq. 1 . The potential fields of Argon, Helium, and graphite are modeled by 6-12 Lennard-Jones potentials as,

$$
U\left(r_{i j}\right)=4 \epsilon\left[\left(\frac{\sigma}{r_{i j}}\right)^{12}-\left(\frac{\sigma}{r_{i j}}\right)^{6}\right]
$$

where $\epsilon$ is a parameter characterizing the strength of interactions, $\sigma$ is the molecular diameter, and $r_{i j}$ is the distance between molecules $i$ and $j$. Lennard-Jones parameters used in the simulations are listed in Table. 1. 
Table 1: Lennard-Jones parameters of Argon, Carbon, and Helium.

\begin{tabular}{|l|l|l|}
\cline { 2 - 3 } \multicolumn{1}{c|}{} & $\epsilon(\mathrm{Kcal} / \mathrm{mol})$ & $\sigma\left({ }^{\circ} \mathrm{A}\right)$ \\
\hline Ar-Ar & 0.2403 & 3.405 \\
\hline C-C & 0.0684 & 3.407 \\
\hline Ar-C & 0.1282 & 3.406 \\
\hline He-He & 0.0218 & 2.640 \\
\hline He-C & 0.0387 & 3.023 \\
\hline
\end{tabular}

The OPLS all-atom force field is used to simulate the flow of Methane molecules in nanochannel (Jorgensen et al., 1996). The charges of the Hydrogen and Carbon atoms of Methane are set to $0.06 e$ and 0.24 $e$, respectively, and the Lennard-Jones parameters are $\sigma_{H}=2.5^{\circ} \mathrm{A}, \epsilon_{H}=0.03 \mathrm{Kcal} / \mathrm{mol}, \sigma_{C}=3.5^{\circ} \mathrm{A}$, $\epsilon_{C}=0.066 \mathrm{Kcal} / \mathrm{mol}$. A cut-off distance of $9^{\circ} \mathrm{A}$ is considered. The wall temperature is set to $300 \mathrm{~K}$ and the location of its carbon molecules is kept fixed using a spring force. An initial Gaussian velocity distribution with a temperature of $300 \mathrm{~K}$ is considered for the gas molecules and the system's energy is minimized to reach the equilibrium in NVT ensemble (constant number of molecules, constant volume, and constant temperature). A time step of $5 f s$ ( $10^{-15}$ seconds) and $2.5 f s$ is considered to perform the time integration of equation of motion with Verlet algorithm for Argon (and Helium) and Methane, respectively. In order to induce a constant pressure gradient across channel, driving forces of 0.07 to $0.35 p N\left(10^{-12}\right.$ Newtons) is considered and applied only to the molecules located in the first $3.5 \mathrm{~A}$ of the nanochannel (inlet region in Fig. 1). The exerted lateral force, $F$, to the molecules is proportional to pressure drop as described by (Zhu et al., 2002; Carr et al., 2011),

$$
\Delta P \propto \frac{N F}{A},
$$

where $A$ is the area perpendicular to the applied force. Determing the actual pressure gradient in NEMD simulations is not straightforward. Therefore, to compare the molecular fluxes and velocities in different pressure gradients, the term $N F / A L$ which is proportional to pressure gradient is used. The number of molecules at the inlet region, $N$, is calculated at each time step and averaged over the total simulation time.

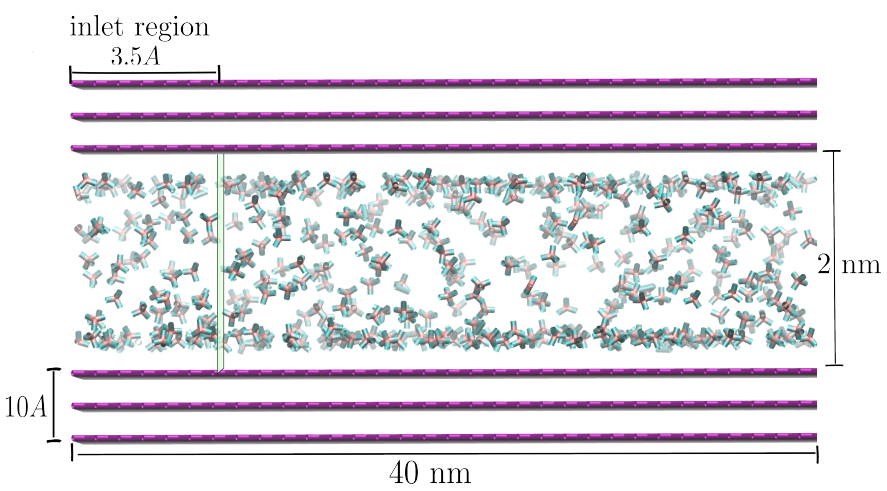

Figure 1: Molecular dynamics simulation geometry. The figure is not in scale.

The fluid temperature is fixed at $300 \mathrm{~K}$ using Berendsen thermostat. The damping coefficient of Berendsen thermostat is set to $2 \mathrm{ps}^{-1}$. The simulations are performed for 3 millions time steps which equals to a total simulation time of 15 nanoseconds $(n s)$. All the simulations are performed using Large-scale Atomic/Molecular Massively Parallel Simulator (LAMMPS) (Plimpton, 1995) and Visual Molecular Dynamics (VMD) (Humphrey et al., 1996) is used for the visualizations. 


\section{Results and Discussion}

\subsection{Equilibrium}

\subsubsection{Adsorption isotherms}

Due to the existence of the attraction forces between the fluid and wall molecules, there is a positional dependence of the fluid density that causes a heterogeneous fluid distribution within the channel. In order to differentiate between the bulk and adsorbed molecules, two quantities are generally defined: Absolute and excess adsorption. Absolute adsorption is the quantity of the gas in the adsorbed state and excess adsorption is the subtraction of adsorbed gas density at the wall and the gas density in the bulk phase.

Absolute and excess adsorption isotherms for the gases in 2 and $4 \mathrm{~nm}$ channels at $300 \mathrm{~K}$ are shown in Fig. 2. The absolute adsorption of all three gases increases as the pressure increases and tend to saturate at high pressures. Methane and Argon show similar absolute loadings, while Helium have lower absolute adsorbed amount. The absolute loadings at the different channel sizes are similar for the pressures simulated.

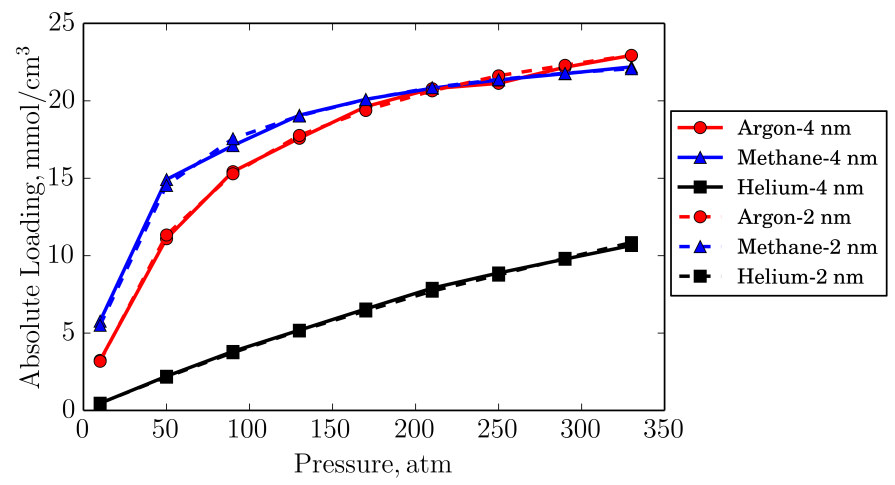

(a) Absolute adsorption

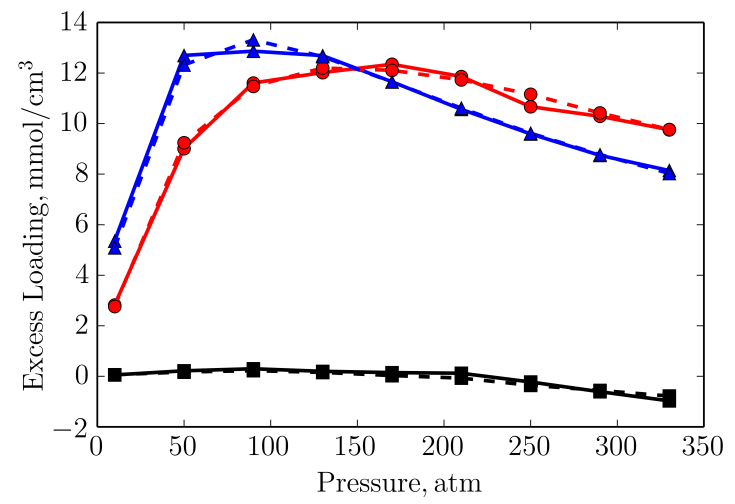

(b) Absolute adsorption

Figure 2: Adsorption isotherms of Argon, Methane, and Helium at 2 and $4 \mathrm{~nm}$ height channel at $300 \mathrm{~K}$. (a) Absolute adsorption (b) Excess adsorption

As pressure increases, the excess loadings increase to reach a maximum value at an optimum pressure and then decreases. This optimum pressure is smaller for Methane than Argon. The maximum excess adsorption for Argon and Methane are 12.2 and $13.3 \mathrm{mmol} / \mathrm{cm}^{3}$, respectively (Fig.2). Helium excess adsorption is almost zero and becomes negative for higher pressures ( $\mathrm{P}>210 \mathrm{~atm})$. This means that the density of Helium at the wall is equal or less than its density in the bulk phase, which is similar to the results presented by Keller and Staudt (2005).

\subsection{Force-Driven Flow}

Force-driven MD simulations are performed for two Knudsen numbers of 0.1 and 0.2 for Argon, Methane, and Helium for four different graphite channel sizes of 2, 4, 6, and $8 \mathrm{~nm}$. Different forces are applied to the molecules at inlet region of the channel (Table. 2). In the following subsections, the velocity profiles, molecular fluxes, and diffusion coefficients of Argon, Methane, and Helium are investigated. 
Table 2: Applied forces to the inlet region of the channels for different gases at Knudsen numbers of 0.1 and 0.2 .

\begin{tabular}{|c|c|c|}
\multicolumn{1}{c|}{} & \multicolumn{2}{c}{ Force $(p N)$} \\
\cline { 2 - 3 } \multicolumn{1}{c|}{} & $\mathrm{Kn}=0.1$ & $\mathrm{Kn}=0.2$ \\
\hline \multirow{3}{*}{ Argon } & 0.139 & 0.208 \\
\cline { 2 - 3 } Methane & 0.208 & 0.278 \\
\cline { 2 - 3 } & 0.278 & 0.347 \\
\hline \multirow{3}{*}{ Helium } & 0.07 & 0.139 \\
\cline { 2 - 3 } & 0.139 & 0.208 \\
\cline { 2 - 3 } & 0.208 & 0.278 \\
\hline
\end{tabular}

Table 3: Pressures for each simulation setup.

\begin{tabular}{|c|c|c|c|c|c|c|}
\hline \multirow{2}{*}{$\begin{array}{c}\text { Size } \\
(\mathrm{nm})\end{array}$} & \multicolumn{6}{|c|}{ Pressure (atm) } \\
\cline { 2 - 7 } & \multicolumn{3}{|c|}{ Kn=0.1 } & \multicolumn{3}{c|}{ Kn=0.2 } \\
\cline { 2 - 7 } & Argon & Methane & Helium & Argon & Methane & Helium \\
\hline 2 & 430 & 240 & 1000 & 195 & 120 & 380 \\
\hline 4 & 190 & 135 & 380 & 97 & 72 & 175 \\
\hline 6 & 127 & 93 & 240 & 58 & 44 & 113 \\
\hline 8 & 99 & 72 & 175 & 50 & 40 & 85 \\
\hline
\end{tabular}

\subsubsection{Pressure calculation}

Determining the exact pressure values in nano-scale channels is challenging in NEMD simulations. However, in GCMC simulations, the pressure can be kept at specific values by the insertion and deletion of the fluid molecules. In order to find the pressure values in the NEMD simulations, a relationship is established between the average gas pressure and the average gas density for each gas at each channel size based on the GCMC simulation results. As an example, the relationship between the average pressure and density of Argon for a 2-nm channel is demonstrated in Fig. 3. Pressure values at different channel heights for Argon, Methane, and Helium are listed in Table. 3.

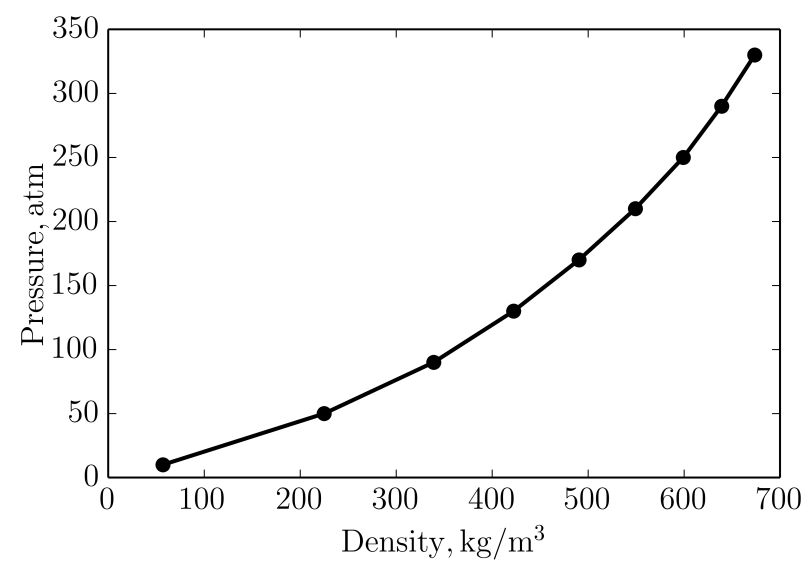

Figure 3: Relationship between the average density and pressure of Argon for the 2- $n m$ channel. 


\subsubsection{Velocity and Density Profiles}

The simulation results of velocity and density profiles of Methane, Argon, and Helium are presented in Figs. 4, 5, and 6, respectively. For each channel height and gas type, the results are plotted for two Knudsen numbers of 0.1 and 0.2 . The velocity profiles are shown with solid blue lines for $K n=0.2$ and solid red lines for $K n=0.1$. The density profiles are shown with blue and red dashed lines for Knudsen numbers 0.2 and 0.1 , respectively.

The bulk and adsorbed densities of each gas decreases as the channel height increases for each Knudsen number. This is due to the fact that as channel height increases, the number density decreases to maintain the same Knudsen number for all channel sizes (Eq. 1). The densities of Argon and Methane at the wall (and similarly average density across the channel height) do not decrease linearly with increasing the channel height. For instance at $K n=0.1$, the average density of Methane across the channel height decreases from $185 \mathrm{~kg} / \mathrm{m}^{3}$ in 2-nm channel to $53 \mathrm{~kg} / \mathrm{m}^{3}$ in 8-nm channel. Similarly, average density of Argon decreases by 3.9 times (from 642 to $164 \mathrm{~kg} / \mathrm{m}^{3}$ ) from the 2 -nm to 8 -nm channel.

To compare the velocities of a gas at different Knudsen numbers, same pressure drop is applied. In NEMD simulations, pressure gradients are proportional to number of molecules and the force applied over the channel length (Eq. 5). For any channel size, the number of molecules for a simulation with $K n=0.1$ is twice those in simulations with $K n=0.2$ for each gas. Therefore, the forces applied to the molecules in the simulations with $K n=0.2$ should be twice of the simulation with $K n=0.1$ to have the same pressure gradients applied. In the velocity profiles plotted in Figs. 4 and 5, the applied forces are 0.139 and $0.278 p N$ for $K n=0.1$ and $K n=0.2$, respectively. The applied forces on Helium molecules (Fig. 6) are 0.07 and $0.139 p N$ for $K n=0.1$ and $K n=0.2$, respectively.
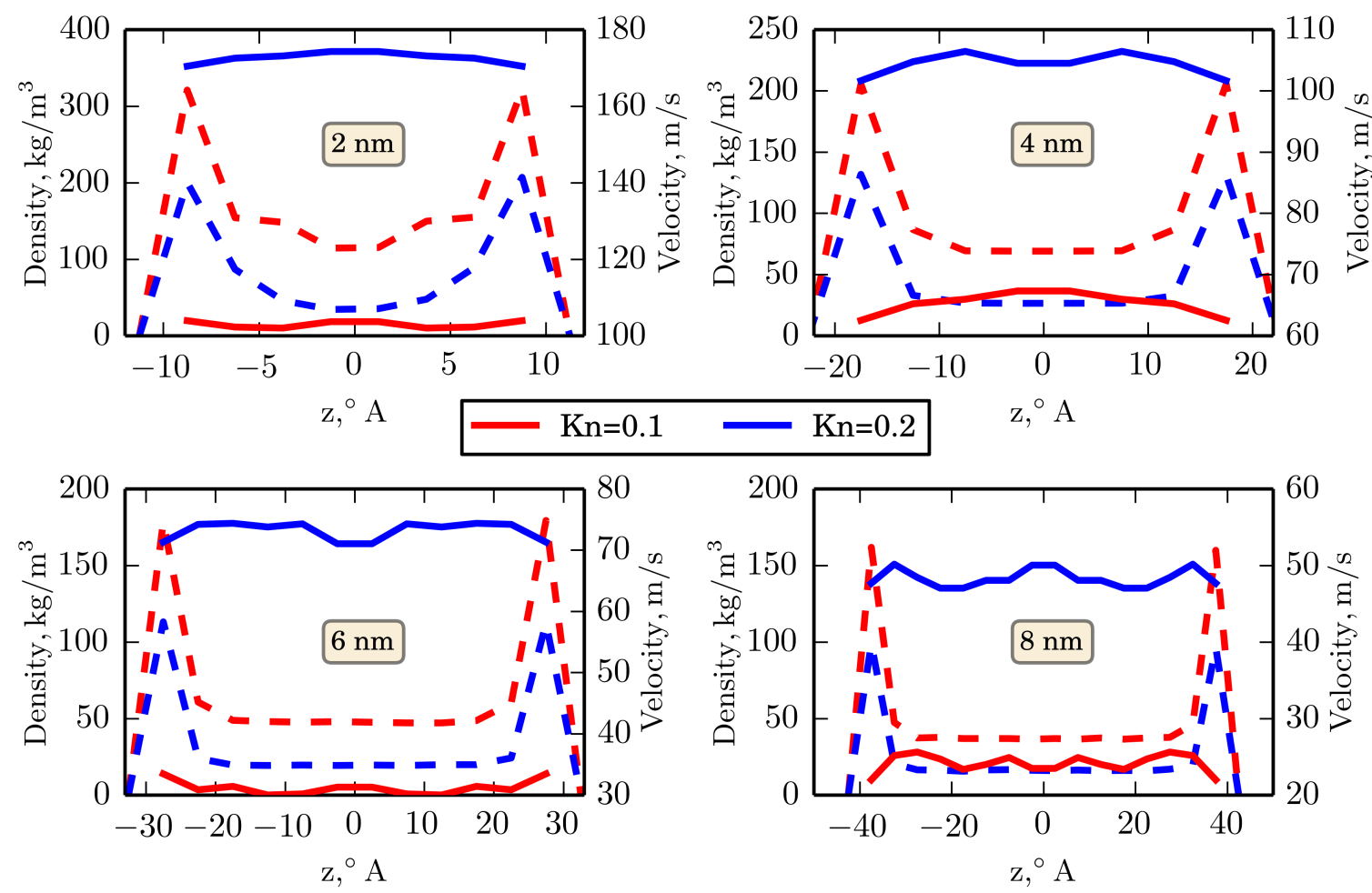

Figure 4: Velocity and density profiles of Methane in the graphite channels. 

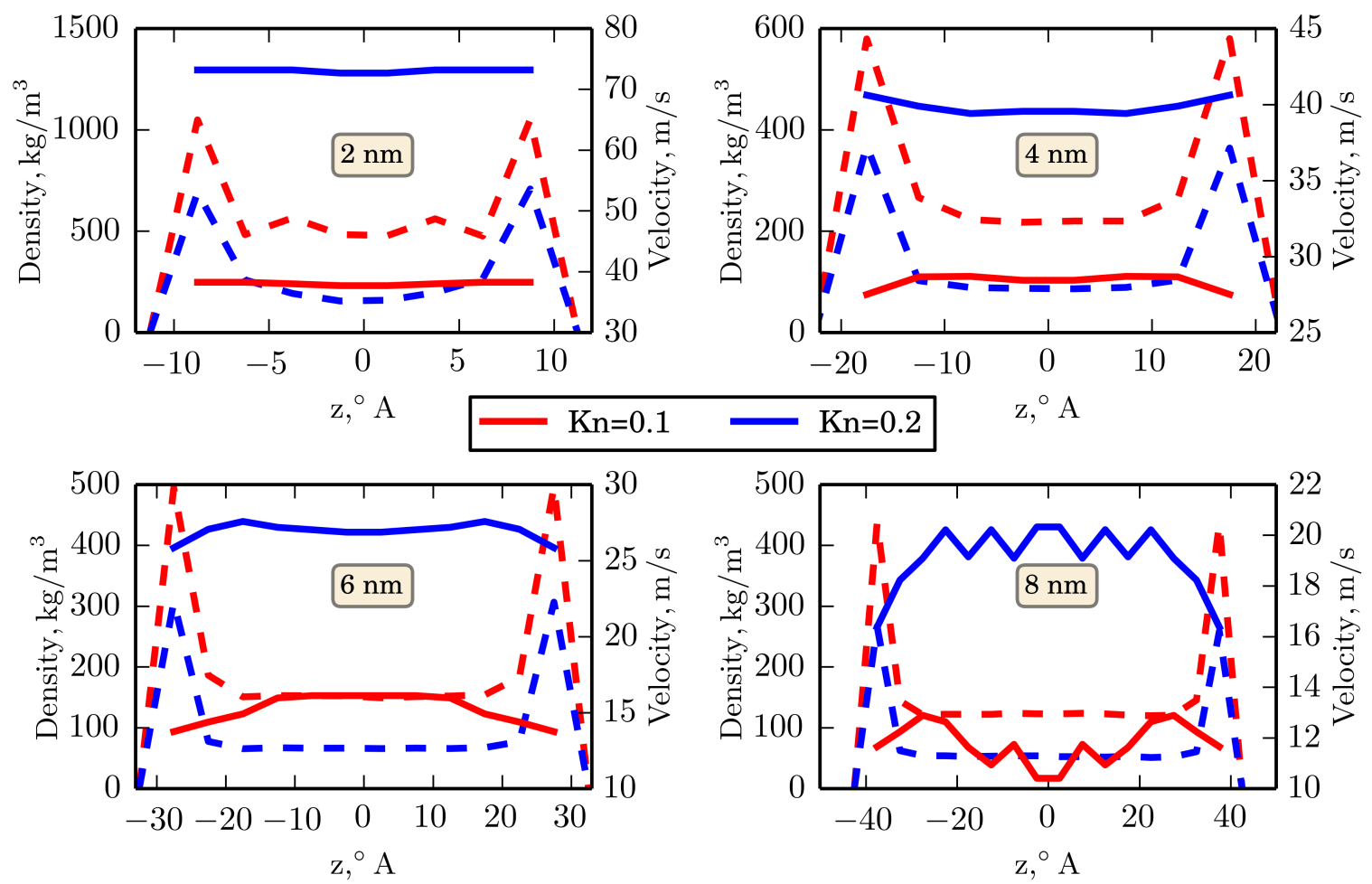

Figure 5: Velocity and density profiles of Argon in the graphite channels.
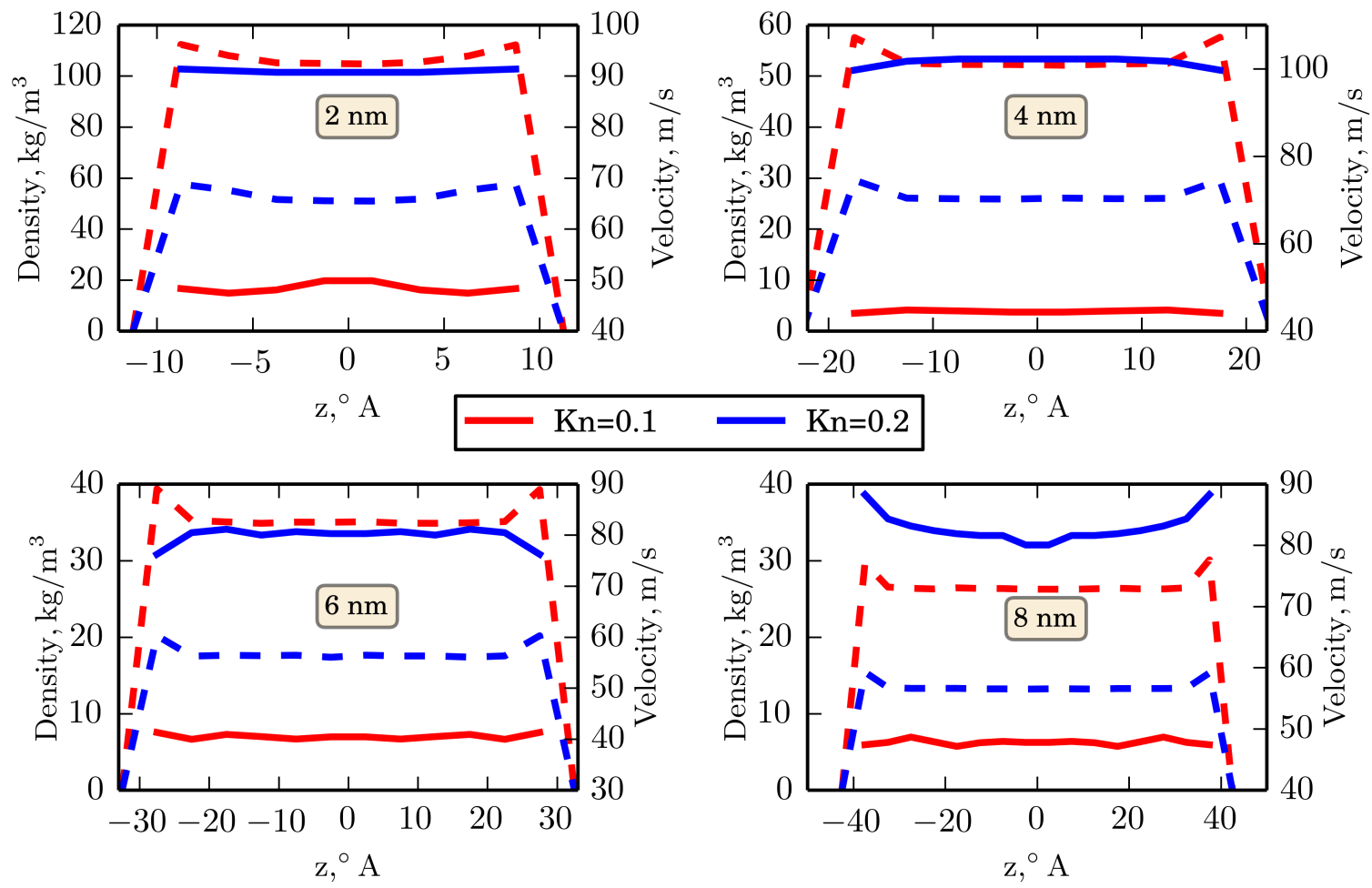

Figure 6: Velocity and density profiles of Helium in the graphite channels. 
For all gases and channel sizes, whether the adsorption affinity is high (Argon and Methane) or low (Helium), plug-shaped velocity profiles are established. In other words, the adsorbed Argon and Methane molecules flow with similar velocities as the gas molecules in middle of channel despite their higher density at the wall. Therefore, the adsorbed phase contribute significantly to the flow. Additionally, for a particular channel height, the higher the Knudsen number, the higher the average gas velocity at the same pressure drop. This result is consistent with predictions of mathematical models of flow in nano-scale channels, e.g., R13 moment method (Kazemi and Takbiri-Borujeni, 2015).

\subsubsection{Molecular Fluxes}

The molecular fluxes of Methane, Argon, and Helium are plotted in Figs. 7a, 7b, and 7c against the pressure gradients for all the channel sizes. For comparison purposes, the pressure drop parameter, $\frac{N F}{A L}$, which is linearly proportional to the pressure gradient, is used. The molecular fluxes are calculated by multiplying the average channel densities by the their average velocities.

For all the gases, the molecular flux increases with increasing the pressure gradient for all the channel sizes. Furthermore, for a specific pressure gradient, the molecular flux of the gases decreases as channel the sizes increase. For all the pressure gradients studied, the highest molecular flux is obtained for the 2-nm channel. For channel sizes less than $8 \mathrm{~nm}$, molecular flux values and the trend of their increase with increasing the pressure gradient is approximately the same for Argon and Methane. For the 8-nm channel, although differences are small, molecular fluxes for Methane are higher than those for Argon.

Molecular fluxes for Helium are smaller than those for Argon and Methane for all channel sizes. The molecular fluxes of Argon and Methane are similar to each other but different from Helium. Molecular fluxes increase from 5050 to $38577 \mathrm{~kg} / \mathrm{m}^{2} \mathrm{~s}$ for Argon and from 4498 to $37244 \mathrm{~kg} / \mathrm{m}^{2} \mathrm{~s}$ for Methane as pressure gradient parameter increases from 0.26 to $2.56 \mathrm{psi} / \mathrm{nm}$ for Fig. 7 a. For the same channel heights, the Helium molecular flux increases from 2725 to $9000 \mathrm{~kg} / \mathrm{m}^{2} \mathrm{~s}$ as the pressure gradient parameter increases from 0.35 to $2.78 \mathrm{psi} / \mathrm{nm}$.

According to the results presented in Figs. 7, the molecular flux is approximately a linear function of the pressure gradient for each gas in the channels. Furthermore, for Argon and Methane which have similar adsorption behaviors, the rate of change of molecular flux with respect to the pressure gradient is approximately the same. This shows that the mass transfer through organic nano-scale conduits is essentially diffusive. This has significant implications in reservoir modeling of unconventional resources because the governing equation used in fluid flow through porous media, Darcy's equation, is derived based on the advective flow assump-

tions. To have a realistic model for predicting the recovery of fluids from unconventional resources, the transport equations in organic nanopores should be replaced by the diffusive transport equations. 


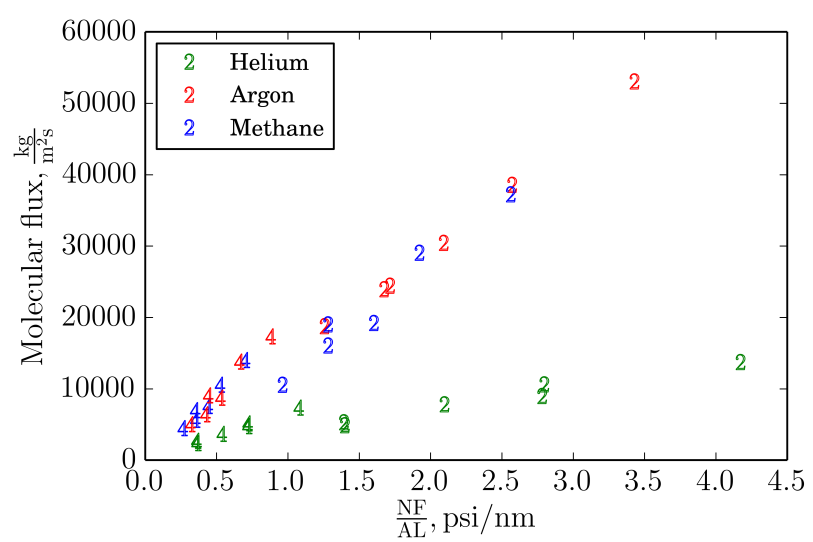

(a) 2 and $4 \mathrm{~nm}$

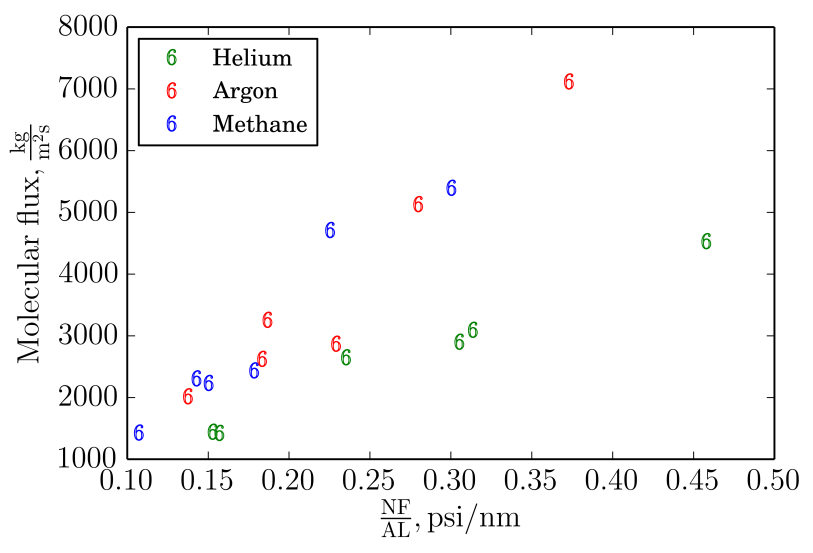

(b) $6 \mathrm{~nm}$

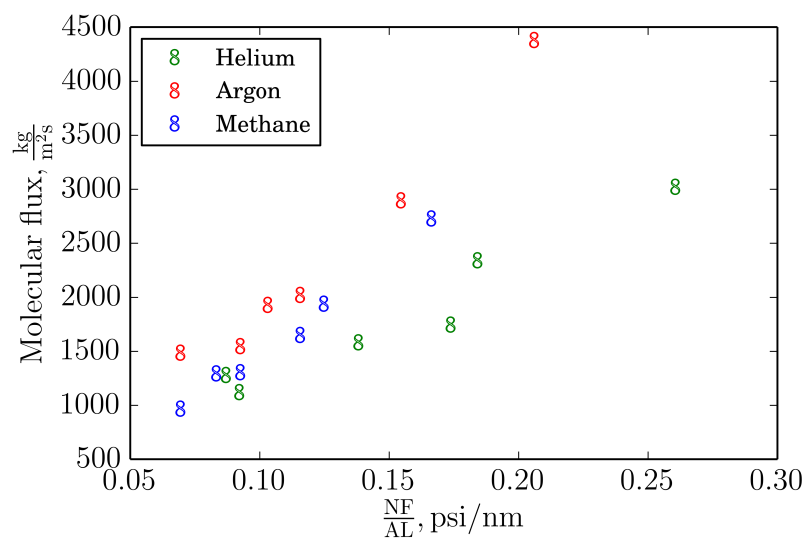

(c) $8 \mathrm{~nm}$

Figure 7: Molecular flux vs. pressure gradient for (a) 2 and $4 \mathrm{~nm}$, (b) $6 \mathrm{~nm}$, and (c) $8 \mathrm{~nm}$.

\subsubsection{Mass Flux Profiles}

In order to compare the mass flux profiles of the gases, the channel is divided to equally-sized bins. At each bin, the average velocity is multiplied by the average density and divided by the product of average velocity and density across the channel height to determine the dimensionless mass flux. The calculated dimensionless mass flux profiles of Argon, Methane, and Helium are plotted for the Knudsen numbers of 0.1 and 0.2 in Fig. 8. Mass flux at the first and last bin, i.e., bins next to the channel walls, have higher dimensionless mass flux values for Argon and Methane compared the ones for the bins away from the walls. This is due to the fact that the density of these two gases are higher near the wall than those of the bulk phase. Mass flux profile of Helium are plug-shaped as the density and velocity values remain approximately constant across the channel height

According to these results, as Knudsen number increases, the contribution of the adsorbed phase to the total mass flux becomes higher. These contributions are $42 \%$ for both Argon and Methane at Kn=0.1, which increases to approximately $53 \%$ at $\mathrm{Kn}=0.2$. 


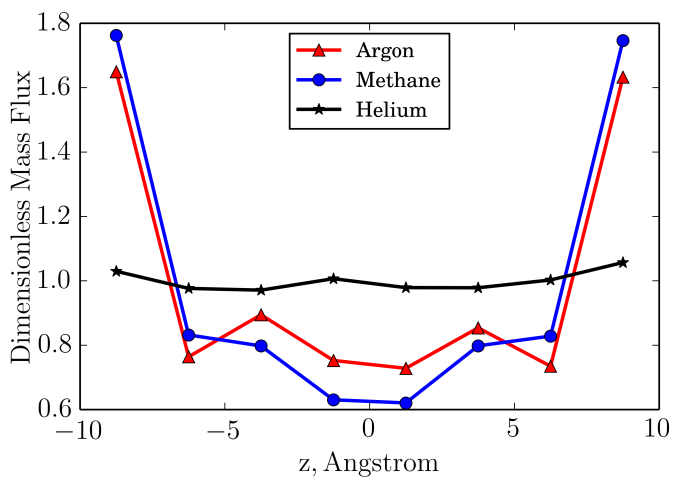

(a) $\mathrm{Kn}=0.1$

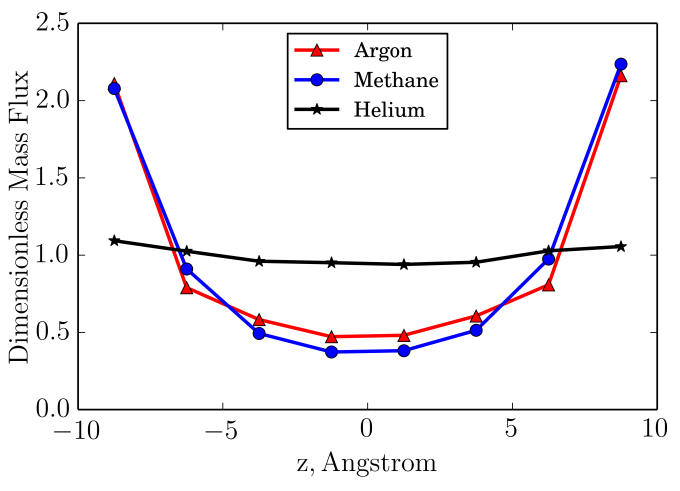

(b) $\mathrm{Kn}=0.2$

Figure 8: Dimensionless mass flux profiles of Argon, Methane, and Helium at (a) Kn=0.1 (b) Kn=0.2.

\subsubsection{Diffusion}

For NEMD simulations, the transport coefficients can be calculated from the measured molecular flux as (Bhatia and Nicholson, 2003),

$$
D_{t}=\frac{k_{B} T j}{\hat{\rho} F}
$$

where $k_{B}$ is the Boltzmann constant, $T$ is the temperature, $j$ is the molecular flux, $\hat{\rho}$ is the average gas density in the channel, and $F$ is the applied external force.

The transport diffusivity coefficients are plotted for Knundsen numbers of 0.1 and 0.2 for Argon, Methane, and Helium in Fig. 9. As mentioned earlier, three different external forces are applied for each channel height at each Knudsen number. The error bars are plotted in Fig. 9 show the variance of diffusion coefficients at different external forces. The transport diffusivity coefficients listed in Table. 4 are the average diffusivity coefficients calculated for the external forces.

For each gas, the highest transport coefficient corresponds to the smallest channel, i.e., $2 \mathrm{~nm}$. For Methane and Argon, transport coefficients decrease as the channel size increases. The diffusion coefficients of Methane are higher than those for Argon for all the channel sizes. For Argon and Methane, the diffusion coefficients are higher at $K n=0.1$ compared with $K n=0.2$ for the same channel size. For Methane, the diffusion coefficients decrease 76 and $70 \%$ as the channel sizes increase from 2 to $8 \mathrm{~nm}$ for Knudsen numbers of 0.1 and 0.2, respectively. For Argon, this decrease is 69 and $72 \%$ for Knudsen numbers of 0.1 and 0.2 , respectively. For Helium, the diffusion coefficients are weak functions of the channel size and Knudsen number. The change in diffusion coefficients for Helium is less that $15 \%$. The average diffusivity coefficients for Helium at $K n=0.2$ are slightly higher than $K n=0.1$ for the same channel size. 
Table 4: Transport diffusion coefficients for Methane, Argon, and Helium at Kn=0.1 and Kn0.2 for all the channel sizes.

\begin{tabular}{|c|c|c|c|}
\hline Gas & Knudsen number & $\begin{array}{c}\text { Channel Height } \\
(\mathrm{nm})\end{array}$ & $\begin{array}{l}\text { Diffusion Coefficient } \\
\qquad\left(10^{-6} \mathrm{~m}^{2} / \mathrm{s}\right)\end{array}$ \\
\hline \multirow{8}{*}{ Methane } & \multirow{4}{*}{0.1} & 2 & 3.06 \\
\hline & & 4 & 1.95 \\
\hline & & 6 & 1.12 \\
\hline & & 8 & 0.732 \\
\hline & \multirow{4}{*}{0.2} & 2 & 2.43 \\
\hline & & 4 & 1.62 \\
\hline & & 6 & 0.969 \\
\hline & & 8 & 0.720 \\
\hline \multirow{8}{*}{ Argon } & \multirow{4}{*}{0.1} & 2 & 1.18 \\
\hline & & 4 & 0.834 \\
\hline & & 6 & 0.474 \\
\hline & & 8 & 0.366 \\
\hline & \multirow{4}{*}{0.2} & 2 & 1.11 \\
\hline & & 4 & 0.622 \\
\hline & & 6 & 0.387 \\
\hline & & 8 & 0.315 \\
\hline \multirow{8}{*}{ Helium } & \multirow{4}{*}{0.1} & 2 & 2.66 \\
\hline & & 4 & 2.75 \\
\hline & & 6 & 2.45 \\
\hline & & 8 & 2.35 \\
\hline & \multirow{4}{*}{0.2} & 2 & 2.85 \\
\hline & & 4 & 2.79 \\
\hline & & 6 & 2.64 \\
\hline & & 8 & 2.46 \\
\hline
\end{tabular}

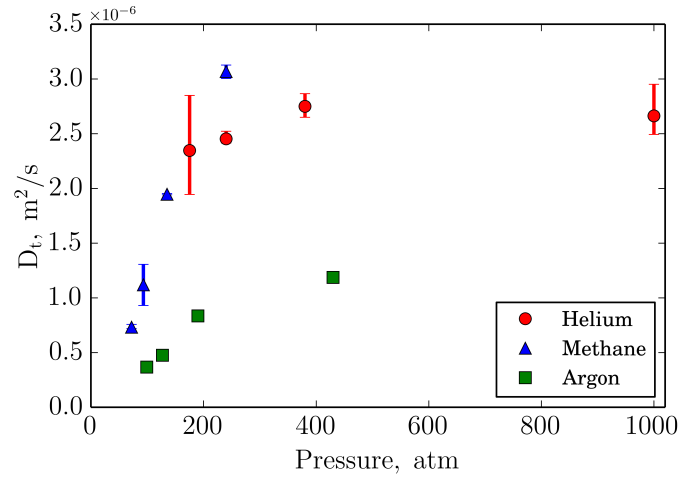

(a) $\mathrm{Kn}=0.1$

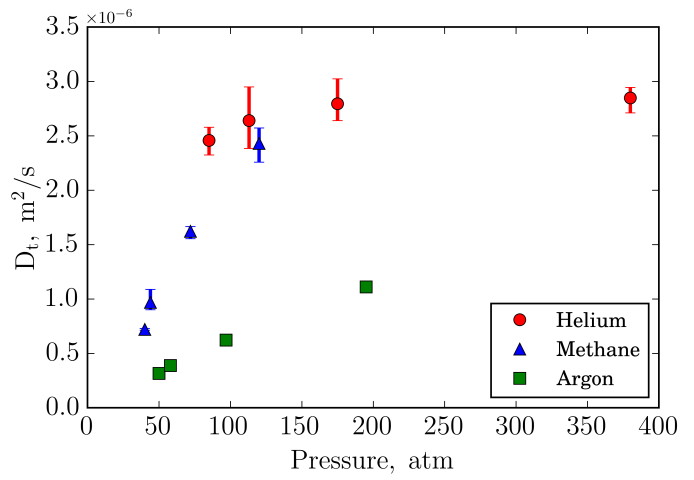

(b) $\mathrm{Kn}=0.2$

Figure 9: Transport diffusion coefficients at (a) $\mathrm{Kn}=0.1$ and (b) $\mathrm{Kn}=0.2$.

\section{Conclusions}

Adsorption and force-driven transport of Helium, Argon, and Methane in four graphite channels of 2, 4, 6 , and $8 \mathrm{~nm}$ hight is studied by performing GCMC and NEMD simulations. Methane and Argon show similar adsorption behavior (absolute and excess adsorptions) while the density of Helium across the channel height 
is approximately constant.

For all gases, whether the adsorption affinity is high (Argon and Methane) or low (Helium), plug-shaped velocity profiles are established for all the channel heights. Therefore, due to the higher adsorbed density values of the Argon and Methane, the adsorbed phase contribute significantly to the overall mass flux across the channel. Mass flux profiles of Argon and Methane across the channels demonstrate a significant contribution of adsorbed molecules to total mass flux. According to these results, as Knudsen number increases, the contribution of the adsorbed phase transport to the total mass flux of the channels becomes higher. This contribution is $42 \%$ for Argon and Methane at Knudsen number of 0.1 , which increases to approximately $53 \%$ at Knudsen number of 0.2 .

For the gases studied, the highest molecular flux is obtained for the 2-nm channel. Molecular flux of Helium is smaller than that of Argon and Methane for all the channel sizes. For channel sizes less than $8 \mathrm{~nm}$, molecular flux values and the trend of their increase with increasing the pressure gradient is approximately the same for Argon and Methane. For the 8-nm channel, molecular flux for Methane is slightly higher than that for Argon.

For each gas, the highest transport coefficient corresponds to the smallest channel, i.e., $2 \mathrm{~nm}$. The diffusion coefficients of Methane are higher than those for Argon for all the channel sizes and they decrease as the channel size increases. For Argon and Methane, the diffusion coefficients decrease as the Knudsen number increases. For Helium, the diffusion coefficients are weak functions of the channel size and Knudsen number.

This study shows that the transport through organic nano-scale conduits is essentially diffusive. This has significant implications in reservoir modeling of unconventional resources because the governing equation used in fluid flow through porous media, Darcy's equation, is derived based on the advective flow assumptions. Therefore, to have a realistic model for predicting the recovery of fluids from unconventional resources, the transport equations in organic nanopores should be replaced by the diffusive transport equations.

\section{Acknowledgements}

Authors acknowledge use of Super Computing System (Spruce Knob and Mountaineer) at WVU, which are funded in part by the National Science Foundation EPSCoR Research Infrastructure Improvement Cooperative Agreement \#1003907, the state of West Virginia (WVEPSCoR via the Higher Education Policy Commission) and WVU.

\section{References}

Arya, G., H. C. Chang, and E. J. Maginn (2001). A critical comparison of equilibrium, non-equilibrium and boundary-driven molecular dynamics techniques for studying transport in microporous materials. Journal of Chemical Physics 115(17), 8112-8124.

Bhatia, S. K. and D. Nicholson (2003). Hydrodynamic origin of diffusion in nanopores. Physical review letters 90(1), 016105.

Carr, R., J. Comer, M. D. Ginsberg, and A. Aksimentiev (2011). Modeling pressure-driven transport of proteins through a nanochannel. Nanotechnology, IEEE Transactions on 10(1), 75-82. 
Collell, J., G. Galliero, R. Vermorel, P. Ungerer, M. Yiannourakou, F. Montel, and M. Pujol (2015). Transport of Multicomponent Hydrocarbon Mixtures in Shale Organic Matter by Molecular Simulations. Journal of Physical Chemistry C 119(39), 22587-22595.

Feng, F., I. Y. Akkutlu, et al. (2015). Flow of hydrocarbons in nanocapillary: A non-equilibrium molecular dynamics study. In SPE Asia Pacific Unconventional Resources Conference and Exhibition. Society of Petroleum Engineers.

Firouzi, M., K. Alnoaimi, A. Kovscek, and J. Wilcox (2014). Klinkenberg effect on predicting and measuring helium permeability in gas shales. International Journal of Coal Geology 123, 62-68.

Firouzi, M. and J. Wilcox (2012). Molecular modeling of carbon dioxide transport and storage in porous carbon-based materials. Microporous and Mesoporous Materials 158, 195-203.

Humphrey, W., A. Dalke, and K. Schulten (1996). Vmd: visual molecular dynamics. Journal of molecular graphics 14(1), 33-38.

Jin, Z. and A. Firoozabadi (2015). Flow of methane in shale nanopores at low and high pressure by molecular dynamics simulations. The Journal of chemical physics 143(10), 104315.

Jorgensen, W. L., D. S. Maxwell, and J. Tirado-Rives (1996). Development and testing of the opls allatom force field on conformational energetics and properties of organic liquids. Journal of the American Chemical Society 118(45), 11225-11236.

Kazemi, M. and A. Takbiri-Borujeni (2015). An analytical model for shale gas permeability. International Journal of Coal Geology 146, 188-197.

Kazemi, M. and A. Takbiri-Borujeni (2016a). Flow of gases in nanocapillary organic nanopores of shale: A boundary-driven molecular simulation study. In SPE Western Regional Meeting. Society of Petroleum Engineers.

Kazemi, M. and A. Takbiri-Borujeni (2016b). Flow of gases in slit shaped organic nanopores of shale: A boundary-driven molecular simulation study. In SPE Low Perm Symposium. Soeciety of Petroleum Engineers.

Keller, J. U. and R. Staudt (2005). Gas adsorption equilibria: experimental methods and adsorptive isotherms. Springer Science \& Business Media.

Plimpton, S. (1995). Fast parallel algorithms for short-range molecular dynamics. Journal of computational physics 117(1), 1-19.

Travis, K. P., K. E. Gubbins, and N. Carolina (2000). Poiseuille flow of Lennard-Jones fluids in narrow slit pores. The Journal of Chemical Physics 112(4), 1984.

Zhu, F., E. Tajkhorshid, and K. Schulten (2002). Pressure-induced water transport in membrane channels studied by molecular dynamics. Biophysical journal 83(1), 154-160. 